\title{
Bias Field Corrected Hippocampus Segmentation using k means Clustering and Region Growing
}

\author{
G.L.N.Murthy ${ }^{1}$ and B.Anuradha ${ }^{2}$ \\ ${ }^{1}$ Dept. of ECE, LBRCE, Mylavaram, Andhra Pradesh, India. \\ ${ }^{2}$ Dept. of ECE, S.V. University College of Engineering, Tirupati, A.P, India
}

\begin{abstract}
Accurate segmentation of critical tissues in Magnetic Resonance (MR) Images is severely affected due to imperfections in the RF coil of the acquisition device. The problem becomes more prominent when there does not exist clear boundaries beaveen various substructures. Hippocampus delineation from given MR image thus requires numerous preprocessing steps for correcting the intensity inhomogeneity resulting due to imperfections. Most of the time, extracted region will be partially contdining the surrounded tissues. In this current work, an effective atgorithm is developed aimed at segmenting the Hippocampus from the human brain $M R$ inage after performing the pre required bias correction. Before the given image is segmented the image contrast is adjusted and then bias field is corrected. These stepsare followed by clustering as well as region growing algorithms to finally yield effective results.
\end{abstract}

Keywords: Bias field, Intensity in Homogeneity, deyel set approach, Gaussian Fit, Clustering

\section{Introduction}

Induced by the abundance of plaques and tangles, the destruction and death of nerve cells causes memory aitures and personality changes. Diagnostically called as Alzheimer's disease (AD), this severe problem affects even the day to day functions of human being. The plaques and tangles are the deposits of Beta Amyloid and Tau, disabling or blocking the communication between nerve cells referred as neurons. These further interupts the processes the cells need to survive. The impact of these deposits, the reasons which are not known exactly is severe atrophy of Hippocampus, a tiny tissue in the human brain. Seyeral studies have indicated that in addition to ageing, severe accident to head and genetically disorders are the fundamental reasons for development of AD. Much research has been carried out and also continuing to develop automated methods that can aid in medical diagnosis. Several imaging techniques were developed which can able to grasp and display the anatomical variations of human tissues. Magnetic resonance Imaging one of numerous imaging techniques that can reflect the structural variations which can be further used for disease identification and treatment planning.

\footnotetext{
The fact behind developing automated algorithms is to reduce the large amount of time and man power needed to identify the problem. Further, with large amount of images available, it will be difficult for a human rater to exactly perform the desired task. Even there is always a possibility of inter rater and intra rater variability. Thus, the developed algorithms concentrate on isolating the Hippocampus from its neighbors and quantifying the atrophy occurred to the neurodegenerative action. However when it comes distinguishing various tissues, the methods developed must consider various parameters that affects the accuracy. The situation may become even worse due to the characteristics that are specific to Hippocampus. The unclear boundaries with neighboring tissues and low contrast always make task of delineating tough.
} 
In the current paper, an effective algorithm is developed which inherits the underlying concepts of Level sets as well as k means clustering. Section II presents an overview of numerous approaches that were defined earlier towards reducing the smooth variations of intensity within the tissue itself. It also describes the methodology used in this work for bias filed correction. Section III elaborates the subsequent steps required to extract the most sought tissue in human brain, Hippocampus that is needed in cognitive related disorders. In section IV a detailed analysis of results obtained was presented.

\section{Preprocessing}

Extracting an object from a population seems an easy task. When it comes to an MR image situation is quite difficult due to the presence of the problem called as intensity in homogeneity (IIH). Elaborated as slow and non-anatomic intensity variation of same tissue, IIH is usually complex to notice by a human observer. However many, segmentation and registration algorithms are capable of sensing unwanted varjations of image intensities.

Bias field is a low frequency and smoothly varying signal that corrupts the MR Images. Satisfactory results cannot be produced when such images are processed for segmentation and analysis. The bias field corrupts the MR images due to intensity in homogeneity of MRI machine. The image is blurred and high frequency contents like edges and contours are affected. The consequence of this is variation in the distribution of pixel intensities within the same tissue.

This smooth variation of tissue intensities (n) MR images is particularly due to the imperfections in the RF coil of the acquistion device as well as RF penetration and standing-wave effects. Manual discrimination of tisøes is not much affected by this variation. However when it is desired to go for automated algorithms for information extraction, this artifact reduces the efficiency of qualitative and quantitative analysis. Even though the non-uniformities due to coil defects can be eliminated, Classical methods cannot significantly reduce the same due to patient anatomy.

The bias field is generally considered to be a slowly spatially varying function and assumed to be multiplicative, consistently with the intrinsic nature of the corrupting physical processes. From the physic of imaging in a variety of modalities (e.g. camera and MRI), an observed image can be modeled as $\mathrm{S}=\mathrm{bI}+\varepsilon$ where $\mathrm{I}$ is the true image, $\mathrm{b}$ is the component that accounts for the intensity inhomogeneity, and $\varepsilon$ is additive noise. The component $b$ is referred to a bias field (or shading image). The true image represents an intrinsic physical property of the objects being imaged.

This artifact can besimply reduced by dividing the corrupted image with an estimate of the back ground. Processing the given image with a low pass filter can yield an approximation of back ground, which however difficult as it is very complex to design a low pass filter with sharp cut off.

An augmented filtering scheme, referred as anisotropic filtering was proposed in [1] is simply capable of processing both $3 \mathrm{D}$ and multi echo MR data. The prominence of this approach is that objective contour as well as boundaries between tissues are not only preserved but enhanced. Further, the diffusion process is a fundamental requirement for segmentation. An iterative algorithm that does not rely tissue intensity model is proposed by Steed in [2]. In this, the data available itself is used in deriving a non-parametric model of intensities. Along with properly choosing the smoothness of the estimated filed as well as the width of the kernel, it needs foreground to be segmented from back ground.

Whenever the segmentation is based on the intensity, an estimate of the inhomogeneity as defined in [3] is better used. It effectively utilizes the polynomial model of the inhomogeneity field with the advantage of independency of image characteristics as well as pre segmented image. A robust and fat optimization process is presented in [4] whose 
energy function combines both local and global criteria. In this, a polynomial approximation of bias field which minimizes composite energy function is developed.

Smooth variations or shifts with in homogeneity regions neither are used designing non parametric model given in [5]. Neither optimization nor is prior tissue identification needed in this. Various approaches for either reducing or completely eliminating Non uniformity were reviewed in [6]. It states that the works on correcting the most typical problem can be seen from long ago back to 1980.Based on studies conducted, sources for inhomogeneity can be either the properties of MRI device or the characteristics of the imaging object itself. Among the both, the second one is more difficult to deal with. The artifacts resulting due to either of the sources can be removed or corrected by using prospective as well as retrospective methods. Prospective methods based on time averaging or multiple scans of the imaging object, concentrate on improvement of image acquisition process.

A variant of the popular nonparametric non uniform Intensity normalization (N3) algorithm is proposed for bias field correction in [7]. An iterative optimization scheme which, among improves convergence performance is developed in this. Adhikari has well developed an approach in [8] that neither requires atlas support nor any reference points. Even though many of the algorithms developed concentrated on correcting intensity in homogeneity, an insight into proper selection of input parameters for optimum correction was given in [9]. After thoroughly, analyzing various indirect metrics, coefficient of Joint Variation (CJV) is identified as the best metric which in urm used to select input parameters for inhomogeneity correction.

In the current work, a level set based approach after appopiate contrast adjustment is used for bias field correction. Even though Level set methods are meant for tissue classification, they can further be extended for correcting the intensity non uniformity. The pixel intensities in each class afe replaced with mean intensity of respective class. This results in a corresponding bias free image. The development of this method has its foundations as proposed in [10] It eoncentrates on tracking the motion of a front whose speed depends on local curvature.

Slowly varying bias fiete as well as the true image being be a constituent on $\mathrm{N}$ distinct images are the assumptions made in [1Y] for achieving segmentation and simultaneous bias correction. A level set function is a function used for represent an image domain I into two disjoin regions R1 and R2.Figure 1(a) shows an image with intensity in homogeneity. After local intensity clustering and bias field estimation, resultant image is shown in 1(b).Two assumplons of slowly varying bias field as well as image is composed of $\mathrm{N}$ values in numerou disjoint regions are made. The underlying mathematical concepts are having their toots in [12].

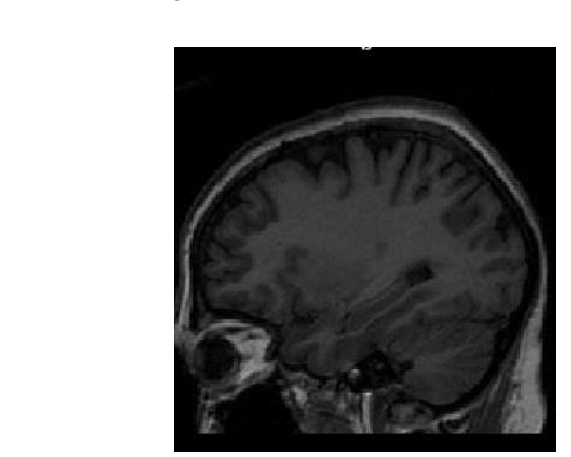

Figure 1. (a) Original Image

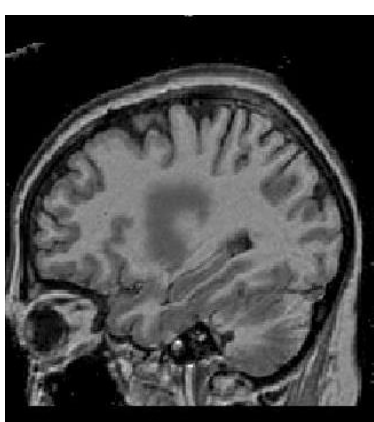

(b) Bias Corrected Image 


\section{Segmentation}

Whenever it is required segment the human brain MR image for disease identification the required preprocessing is carried out as mentioned in the previous section. In particular many clinical applications like Epilepsy Alzheimer's Disease (AD) as well as Mild cognitive impairment utilize shape analysis of Hippocampus as an important bio marker.

Several automated segmentation algorithms were proposed earlier to effectively extract the Hippocampus. These algorithms are however complex as they either need a model to be prepared based on prior knowledge of the object of interest or involve a variety of steps as required in atlas based methods. Even in atlas based methods, much of the computations were involved particularly in the preparation of atlas. This is significant as a single atlas cannot yield satisfactory results as MR images can be of different age groups as well as different imaging modalities.

Geometric properties and statistical characterization of normal shape variations were used for quantification of shape and size of hippocampus in [12].However it requires manual definition of number of points along the boundary of hippocampus. Carmichael has utilized registration methods in [13] which produced geometric deformation of high degree. These deformations are subsequently used for automated segmentation and resulted in higher degree of argument with manual segnentation. Hippocampus extraction based on land mark localization is presented in [14].B ased on the traming set supported by numerous land marks Gaussian models were evolved which are eapable of structure identification.

A novel algorithm with reduced yolme differences within the population was proposed in [15]. The significance of the algorithm is hat Intra Correlation Coefficient and Similarity index are better than molti atlas segmentation. Van Leemput[16] has demonstrated a model based approach for extracting the hippocampus. The proposed model has incorporated a prior dist ibution of the occurrence of neuro anatomical models.

$\mathrm{Ni}$ proposed a local histogram based segmentation in [17]. Instead of opting for histogram differentiation, the work considened local histograms as data descriptors. The model proposed defined a partition such that each region contains only similar local histograms.

The similarity between small sub volumes of the image referred as patches, a new label fusion approach has been developed in [18]. It effectively utilizes the underlying properties of non-local means filtering termed as natural redundancy of information and local intensity context. Kim [19] developed an approach for segmenting 7.0 T MR Images based on Auto Contey Models. The simplicity lies here in that the context information between around each voxel required for discriminating hippocampal and nonhippocampal vôlumes is achieved through simple linear registration among atlases.

An algorlthm aimed at reducing the number of atlases without compromising on the segmentation accuracy was presented in [20], which considers a limited number of atlases for generating a template library. Construction of a statistical atlas of hippocampus formation was described in [21]. Various distribution functions were considered to reflect the little contrast variation between various white matter structures. The idea behind the current work is develop an algorithm which cans complexity in the preparation of an atlas or any model of the region to be extracted.

K - Means clustering: It is known that in the process of clustering, a given data set is divided into certain number of groups. The number of regions into which the data set is to be separated depends on the characteristic of the given image. When it is thought of segmenting human brain MRI, the fundamental constituent elements are Gray Matter (GM), White Matter (GM) and Cerebro Spinal Fluid (CSF).As the earlier preprocessing step of bias field correction properly defined the boundaries between various tissues, by applying this clustering the objects of interest can be more easily delineated. If the image 
is of resolution $\mathrm{nXm}$ and $\mathrm{I}(\mathrm{x}, \mathrm{y})$ be the input pixel to be clustered then the following are the steps involved

i) Initialize the number of clusters as well as their centroids

ii) Calculate the Euclidean distance between each pixel $\mathrm{I}(\mathrm{x}, \mathrm{y})$ and cluster center ck, defined as

$$
\mathrm{d} \varepsilon=\|I(x, y)-c k\|
$$

iii) Based on the distance assign the pixels to its nearest center.

iv) Recalculate the new centroid, defined as

$$
c_{k}=\frac{1}{k} \sum_{i \in c_{k}} \sum_{j \in c_{k}} I(i, j)
$$

v) Repeat the steps till final segmentation is over.

An MR image in sagittal section segmented using $\mathrm{k}$ means clusterin is shown in Figure 2. It can be observed that Hippocampus boundaries with nieghbourmg tissues are almost clear. This is the significance of bias filed correction. Further, it is seen that still a trace of overlap still exsiting. The next objective is to elmmate this overlap and finally extract the desired region.

Region growing: The clustered MR slice is passed through simple region growing steps to finally extract the hippocampus. In this arbitrary seed pixels are chosen. Region is grown from the seed pixel by adding in neighbouring pixels that are similar, increasing the size of the region. When the growth of one regionstops, simply choose another seed pixel which does not yet belong to anyregion and start again. This whole process is continued until all pixels belong to some region.

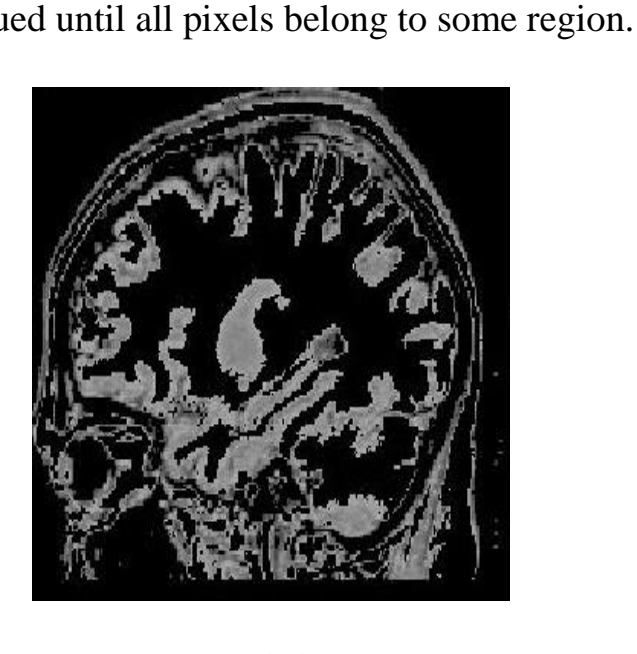

(a)

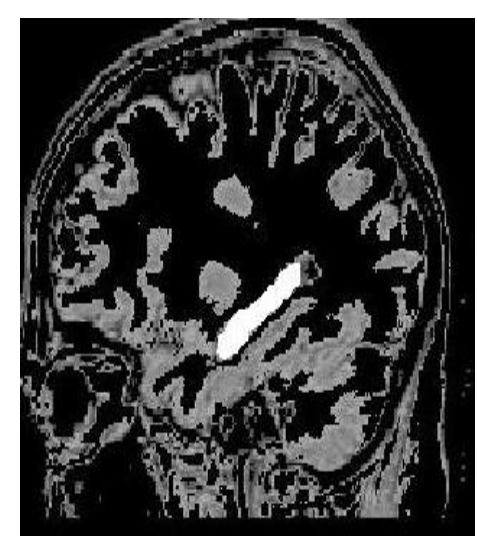

(b) 


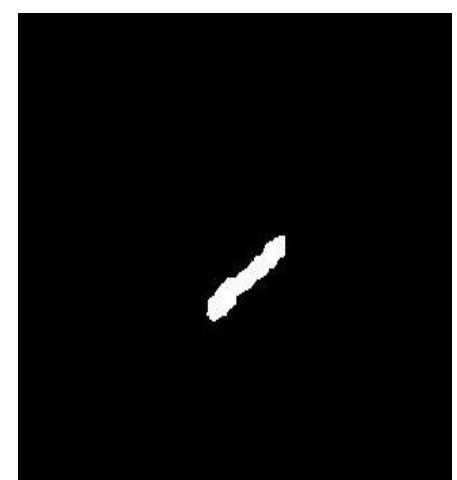

(c)

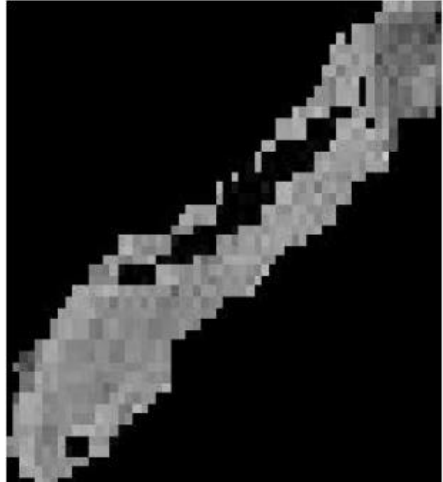

(d)

Figure 2. (a) Clustered Image (b) Region Grown Image (c) Mask Generated (d) Extracted Region

\section{Discussion:}

The proposed algorithm was implemented on 20 MR sagittal view/image data sets. Initially Intensity non uniformity is corrected by using local clustering based level set approach. It was observed that the contrast was well nodified due to which subsequent segmentation steps can be efficiently implemented. The earlier work was image and intensity dependent [22-25]. Any new test image was unable to yield an accurate result which was observed through numerous similanty measures. However, the efficiency of any algorithm should be verified for better performance. Dice and Jaccard coefficient are the two mostly used segmentation performance evaluation measures that were as defined below.

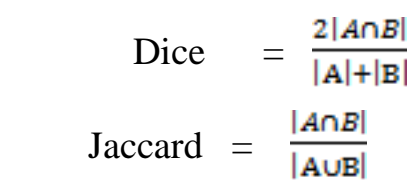

Where $\mathrm{A}$ is the segmentation result obtained through proposed algorithm while $\mathrm{B}$ is the underlying ground truth image. A comparison of the above mentioned parameters for different data sets is shown in Figure 3. The ground truth images were obtained using ITK-Snap. If is observed that a Maximum value of 0.87 was noticed while on average it stood at 0.79 for Dice coefficient. Similarly, better results were shown for Jaccard coefficient with a maximun value of 0.70 and an average value of 0.6 as shown in Figure 3(a). Figure 3(b) denonstrates very lower values of false negatives and false positives are observed which indicates the efficiency of the algorithm. The advantage lies in the fact that without using any complex steps, simple clustering as well as region growing approachês were involved in the segmentation process.
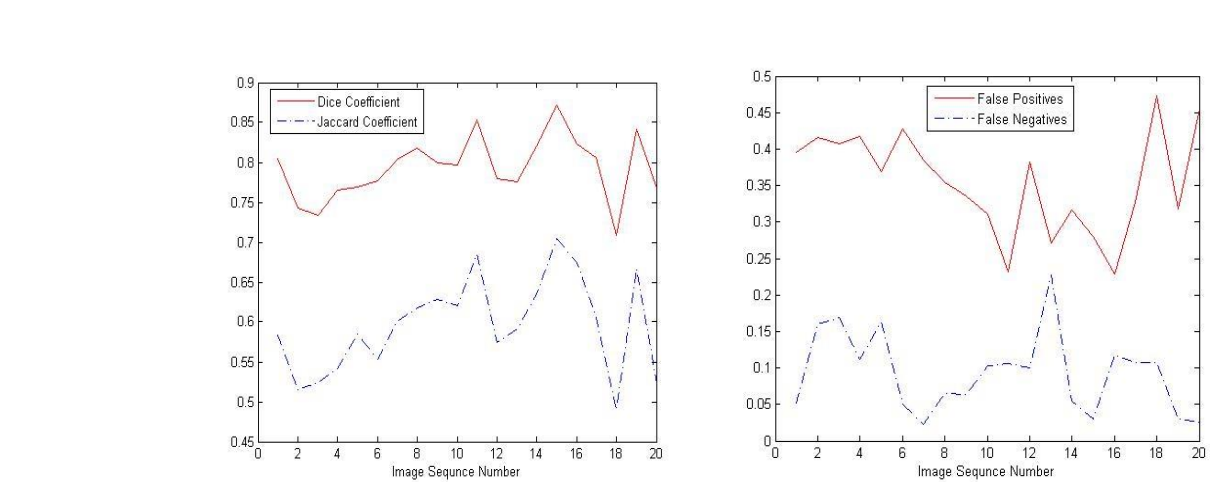

Figure 3. (a)Image Sequence Vs Dice / Jaccard Coefficient (b) Image Sequence Vs False Positives \& Negatives 


\section{References}

[1] G. Gerig, O. Kubler, R. Kikinis and F. A. Jolesz, "Nonlinear Anisotropic Filtering of MRI Data”, IEEE Transactions on Medical Imaging, vol. 11, no. 2 (1992).

[2] J. G. Sled and A. P. Zijdenbos, "A Nonparametric Method for Automatic Correction of Intensity Nonuniformity in MRI Data”, IEEE Transactions on Medical Imaging, vol. 17, no. 1, (1998).

[3] M. Styner, C. Brechbuhler, G. Szekely and G. Gerig, "Parametric estimate of intensity inhomogeneities applied to MRI", IEEE transactions on medical imaging, vol. 19, no. 3, (2000), pp. 153-165.

[4] A. A. Samsnov, R. T. Whitaker, E. G. Kholmovki and C.R. Johnson, "Parametric Method for Correction of Intensity Inhomogeneity in MRI Data", (2002).

[5] E. Vokurka, N. Thacker and A. Jackson, "Diagnostic Radiology, Department of Medicine A Fast Model Independent Method for Automatic Correction of Intensity Non-Uniformity in MRI Data", Journal of Magn Reson Imaging, (1999).

[6] U. Vovk, F. Pernus and B. Likar, "A Review of Methods for Correction of Intensity Inhomogeneity in MRI”, IEEE Transactions on Medical Imaging, vol. 26, no. 3, (2007).

[7] N. J. Tustison, B. B. Avants, P. A. Cook, Y. Zheng, A. Egan, P.A. Yushkevich and J. C. Gee, "N4ITK: Improved N3 Bias Correction", IEEE Transactions on Medical Imaging, vol. 29, no. 6, (2010).

[8] S. Kumar Adhikari, J. Kanta Sing, D. Kumar Basu, M. Nasipuri and P. Kumar Saha, "A nopparametric method for intensity inhomogeneity correction in MRI brain images by fusion of Gaussian surfaces", Signal, Image and Video Processing, vol. 9, issue 8, (2015), pp. 1945-1954.

[9] M. Ganzetti,Wenderoth and D. Mantin, "Intensity Inhomogeneity Correction of Structural MR Images: A Data-Driven Approach to Define Input Algorithm Paranteters in Medical Lnages", Article in Medical Physics, vol. 41, no. 2, (2014), pp.022302.

[10] S. Osher and J. Sethian, "Fronts propagating with curyature-dependent speed: Algorithms based on Hamilton-Jacobi formulations", J. Comp.Phys., vol. 79, no. 1, (1988), pp. 12-49.

[11] C. Li, R. Huang, Z. Ding, J. Chris Gatenby, D. N. Metaxas and J. C. Gore, A Level Set Method for Image Segmentation in the Presence of Intensity Inhomogeneities_With Application to MRI", IEEE transactions on image processing, vol. 20, no. 7, (2011).

[12] T. Chan and L. Vese, "Active contours without edges", IEEE Transactions on .Image. Processing, vol. 10, no. 2, (2001), pp. 266-277.

[13] D. Shen, S. Moffat, S.M. Resnick and C. Daratzikos, "Measuring Size and Shape of the Hippocampus in MR Images Using a Deformable Shape Model", Neurolmage, vol. 15, (2002).

[14] O.T. Carmichael,H.A. Aizenstein , S.W. Davis, J.T. Becker, P.M. Thompson, C.C. Meltzer and Y. Liu, "Atlas-based hippocampus segmentation in Alzheimer's disease and mild cognitive impairment", Neuroimage, (2005).

[15] M.-R. Siadat, H. Soltanian-Zadeh and K V Elsevich, "Knowledge-based localization of hippocampus in human brain MRI", Computers in Biology and Medicine, vol. 37, (2007), pp. $1342-1360$.

[16] F. van der Lijn, T. den Heijer, M. M.B. Breteler, W. J. Niessen, "Hippocampus segmentation in MR images using atlas registration, voxêt classification, and graph cuts", NeuroImage, vol. 43, (2008).

[17] K. Van Leemput, A Bakkour, T. Benner, G. Wiggins, L. L. Wald, J. Augustinack, B. C. Dickerson, P. Golland and B. Fischl ,"Automated Segmentation of Hippocampal Subfields From Ultra-High Resolution In Vivo MRI", Hippocampus, vol. 19, no. 6, (2009), pp. 549-57.

[18] K. Ni, X Bresson, T. Chan and S. Esedoglu, "Local Histogram Based Segmentation Using the Wasserstein Distance", International Journal of Computer Vision, vol. 84, issue 1, (2009), pp. 97-111.

[19] P. Coupé, J. V. Manjón, V. Fonov, J. Pruessner, M. Robles and D. Louis Collins, "Patch-based segmentation using expert priors: Application to hippocampus and ventricle segmentation", NeuroImage, vol. 54, (2011), pp. 940-954.

[20] M. Kim, G. Wu, W. Li, L. Wang, Y.-D. Son, Z.-H. Cho and D. Shen, "Automatic hippocampus segmentation of 7.0 Tesla MR images by combining multiple atlases and auto-context models", Neuroimage, Dec., vol. 83, (2013), pp. 335-345.

[21] . Pipitone, M. T. M. Park, J. Winterburn, T. A. Lett, J. P. Lerch, J. C. Pruessner, M. Lepage, A. N. Voineskos and M. Mallar Chakravarty, "Multi-atlas segmentation of the whole hippocampus and ubfields using multiple automatically generated templates", NeuroImage, vol. 101, (2014), pp. 494-512. J. Eugenio Iglesias, J. C. Augustinack, K. Nguyen, C. M. Player, A. P. M. Wright, N. Roy, M.P. Frosch, A. C. McKee, L. L.Wald, B. Fischl and K. V. Leemput, "A computational atlas of the hippocampal formation using ex vivo, ultra-high resolution MRI: Application to adaptive segmentation of in vivo MRI", NeuroImage, vol. 115, (2015), pp. 117-137.

[23] B. Naresh Kumar Reddy, "Communication energy constrained Spare core on NoC", 6th International Conference on Computing, Communication and Networking Technologies (ICCCNT), (2015), pp. 1-4.

[24] B. Naresh Kumar Reddy, "A Gracefully Degrading and Energy-Efficient Fault Tolerant NoC Using Spare core”, 2016 IEEE Computer Society Annual Symposium on VLSI, pp. 146-151, (2016).

[25] G.L.N. Murthy, B. Anuradha, C.H. Siva Rama Krishna, B. Naresh Kumar Reddy and J.V.N. Ramesh, "Effective utilization of labeling algorithms for Hippocampus segmentation", European Journal of scientific Research, 206-11, vol. 134,no. 2, (2015). 
International Journal of Hybrid Information Technology

Vol. 9, No.12 (2016)

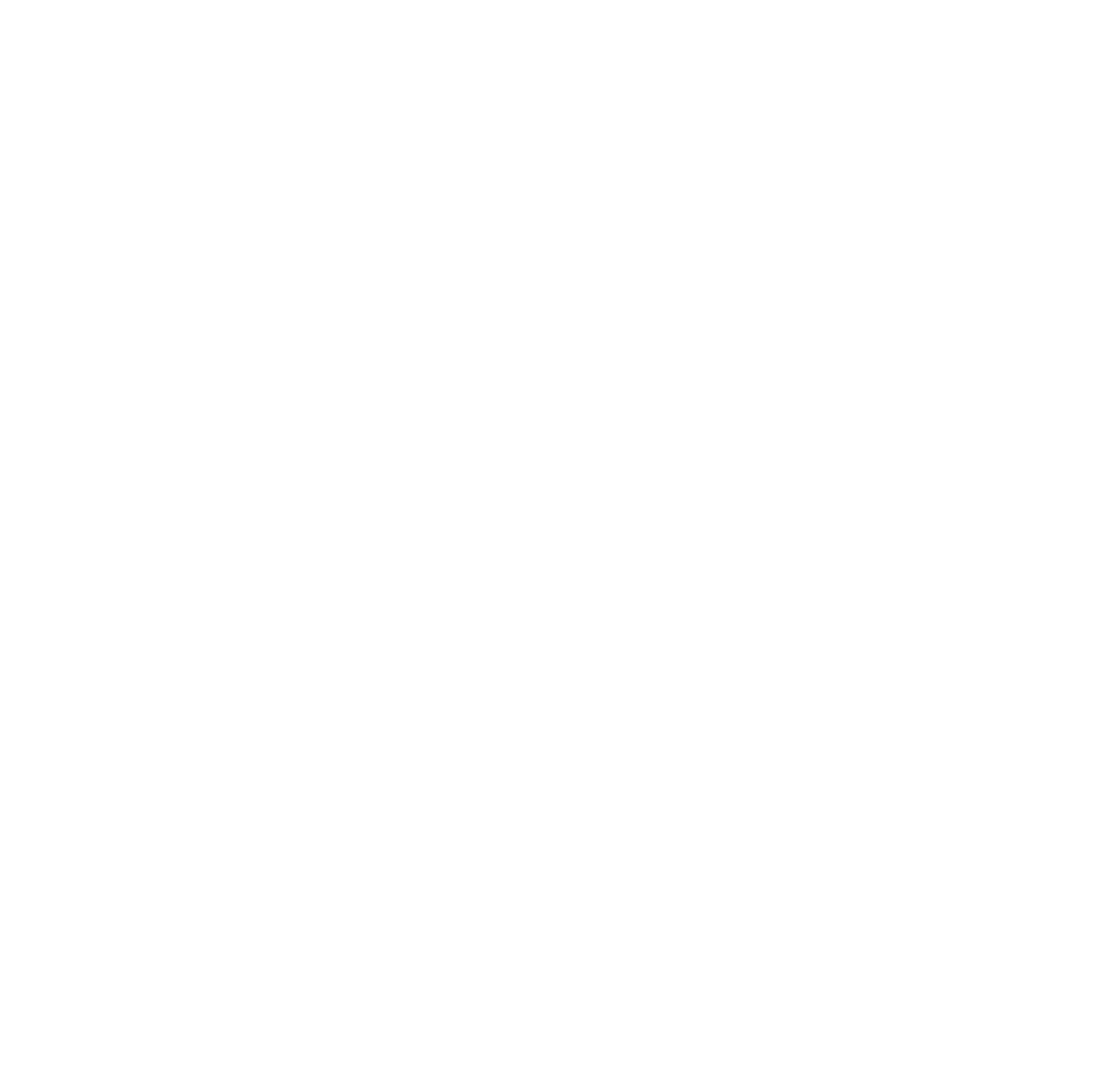

